\title{
Multilevel wrApper Verification System with Maintenance Model Enhancement
}

\author{
Nigonda M. Patil ${ }^{1}$, H. P. Khandagale ${ }^{2}$ \\ ${ }^{1,2}$ Computer Science and Technology Department, Department of technology, Kolhapur, India
}

\begin{abstract}
The online data sources have prompted to an extended usage of wrappers for extract data from Web sources. We introduced a unique idea, to explain the expressed problems and formally demonstrate its accuracy. Conventional research techniques have focused on snappy and effective era of wrappers; the advancement of devices for wrapper support has gotten less consideration and no arrangement to self upkeep. This empowers us to learn wrappers in a totally unsupervised way from consequently and inexpensively preparing information, e.g., utilizing word references and standard expressions. This transforms into a research issue since Web sources frequently change progressively in ways that keep the wrappers from removing data accurately. We will probably help programming engineers develop wrapping operators that translate questions written in abnormal state organized language. Work introduces a proficient idea for auxiliary data about information from positive cases alone. Framework utilizes this data for wrapper upkeep applications: utilizing wrapper check and enlistment component planning a support show. The wrapper verification system identifies when a wrapper is not removing right information, for the most part on the grounds that the Web source has changed its organization. Sites are continuously advancing, upgrading and basic changes happen with no cautioning, which for the most part results in wrappers working incorrectly. Tragically, wrappers may flop in the undertaking of separating information from a Web page. If its structure changes, once in a while even marginally, in this way requiring the mishandling of new procedures to be naturally held to adjust the wrapper to the new structure of the page, in the event of disappointment.
\end{abstract}

Keywords: Wrapper, reinduction, verification, Web Source, Information Extraction

\section{Introduction}

Wrappers are utilized as a part of a considerable measure of genuine situations as enterprise data combination, setting mindful advertising, database building, business insight and focused insight, practical web application testing, supposition mining, or reference databases It is all around perceived that the Web is a profitable wellspring of data and that making utilization of its information is an extraordinary chance to make learning with both logical and business suggestions. The lead above not just takes a shot at this specific site page, it deals with any page created by this frame. Such a run is known as a wrapper, and the issue of inciting wrappers from named cases has been broadly contemplated.

Wrappers are little utility of program used to focus data from sites and structure them for further application handling. Sites are ceaselessly redesigning and basic changes happen with progressively, which generally brings about wrappers working erroneously. In this manner, wrappers support is important for identifying whether wrapper is extricating unessential information. The arrangement utilizing confirmation and support models to distinguish whether wrapper yield is measurably like the yield delivered by the wrapper itself when it was effectively summoned before. Current framework show a few disadvantages, as the information used to assemble these models should be homogeneous, free or delegate enough. In this work, utilize Maintenance Base MAVE, a novel multilevel wrapper check framework that depends on one class order procedures to beat past majors. The exploratory results demonstrate that our proposition beats exactness of current arrangements.

The expansion of online data sources has prompted to an expanded utilization of wrappers for separating information from Web sources. The past research has concentrated on speedy and productive era of wrappers; the improvement of apparatuses for wrapper upkeep has gotten less consideration. This is a critical research issue since Web sources frequently change in ways that keep the wrappers from extricating information accurately. Here present a proficient calculation that takes in basic data about information from positive illustrations alone. Additionally, how this data can be utilized for two wrapper support applications: wrapper confirmation and re-induction. The wrapper check framework finds when a wrapper is not removing right information, for the most part in light of the fact that the Web source has changed its structure. The reinduction calculation naturally upgrades from changes in the Web source by perceiving information on Web pages so that another wrapper might be produced for this source.

There is a huge data accessible on the web, yet a lot of this data is designed to be effectively perused by human clients, not PC applications. Extricating data from semi organized Web pages is an inexorably essential issue for Web-based programming applications that perform data administration capacities, for example, shopping operators and virtual travel associates these applications, frequently alluded to as specialists, depend on Web wrappers that focus data from semi-organized sources and change over it to an organized arrangement. Semi-organized sources are those that have no expressly indicated syntax or blueprint, yet have a certain sentence structure that can be utilized to recognize important data on the page. Indeed, even content sources, for example, email messages have some structure in the heading that can be abused to separate the date, sender, recipient, title, and body of the messages. Different sources, for example, online surveys indexes, have an extremely consistent structure that can be misused to concentrate every one of the information consequently. 


\section{International Journal of Science and Research (IJSR) \\ ISSN (Online): 2319-7064}

Index Copernicus Value (2015): 78.96 | Impact Factor (2015): 6.391

\section{Brief Literature Survey}

In MAVE: Multilevel wrApper Verification system [1] introduce Wrappers are bits of programming used to focus on information from different sites and structure them for further application preparing. Tragically, sites are constantly developing and auxiliary changes happen with no cautioning, which generally brings about wrappers working inaccurately. In [2] Web data extraction, applications and techniques: A survey Wrapper Verification is not a slight undertaking and, on the off chance that it is not performed appropriately, it might build joining costs. In this area, we exhibit the issues that must be adapted to by proposition to perform wrapper confirmation.

Semantic anomaly detection in online data sources [3] Our study of writing rendered a few recommendations to manufacture verifiers. The greater part of them concentrate on a select various predefined components, and score their profiles on an outcome set as a weighted aggregate, which is then contrasted with a predefined edge to figure out if an alert must be flagged or not. Automatic wrapper adaptation by tree edit distance matchy [27] Different proposition give an account of elements and give no way to survey its qualities or to union it with different components (e.g.,[27]). Beyond these straight forward recommendations, we have found a couple others that expand on integrity of-fit or probabilistic techniques.

Wrapper maintenance: A machine learning approach proposed in [4] is relevant the length of the working set used to manufacture the verifier is depicted utilizing an arrangement of numerical elements. The most critical of them is the execution of the DATAPROG calculation, made by similar creators. Generally, the check model is surmised as a vector in which each component is connected with its normal esteem. Later, when another unsubstantiated working set should be checked, they compute another vector in which each element is connected with its normal esteem in the unconfirmed preparing set. In the event that both vector output be considered factually equivalent, the unconfirmed working set is then judged legitimate; else it is invalid. To figure out whether two vectors are factually equivalent, the creators utilize the outstanding 2 integrity off it technique, which thusly depends on Pearson measurement. At that point, the profile of an element essentially figures the comparing numbers to be added of this measurement, and the score registers the measurement itself. Mapping maintenance for data integration systems [9] Change those Pearson fact utilized Eventually Tom's perusing [4]to adjust it of the offers utilized. These writers move forward those comes about attained Toward [4], yet help no data something like those values used to make this transform. Kushmerick devises two closely-related probabilistic systems Previously, both proposals, called

RAPTURE, Kushmerick's ticket might have been building An confirmation model Likewise though features were irregular variables whose circulations camwood a chance to be inferred from An preparing set. Wrapper induction: Efficiency and expressiveness, wrapper Verification[1][13] Thus, a worth of a characteristic On an unsubstantiated preparing set might be deciphered Likewise the likelihood that the quality watched for those comparing characteristic in a authentication model may be steady for those dissemination for the comparing arbitrary variable. Kushmerick proposes utilizing those gaussian appropriation or an experimental appropriation Similarly as probabilistic circulations will profile characteristic values. Kushmerick's score must after that figure those likelihood that all characteristic values would steady with their relating distributions, which Typically obliges analysing the measurable dependencies the middle of Characteristics. The creator proposes utilizing a reliance suspicion Rather. This suspicion may be An capacity that takes An set of probabilities as input, Furthermore returns those comparing joined likelihood. Note that those scores of the preparing situated could Additionally make recognized a test of a irregular variable, which camwood Subsequently a chance to be portrayed Eventually Tom's perusing An probabilistic dissemination too. Wrapper Verification, Mapping maintenance for data integration systems, [13][14] On focus if a alert must a chance to be signalled by virtue of the score of a preparing set, the likelihood that An arbitrary variable takes that worth may be contrasted with An predefined edge that controls how negative confirmation may be (i. E. , those higher the threshold, those less averse an unsubstantiated working situated is adjudged invalid). Dissident is the name of the techno babble portrayed in[14] What's more it takes impulse starting with [13]

DIADEM: Thousands of Websites to a Single Database[19] The web is flooding with verifiably organized information, spread over countless locales, shrouded profound behind pursuit frames, or siloed in commercial centres, just available as HTML. Programmed extraction of organized information at the size of a huge number of sites has long demonstrated subtle, notwithstanding its focal part in the "web of information".

\section{Proposed Work}

An issue that has been largely ignored in previous work on extracting data from web source is that sites change and they change often. The wrapper verification issue by analyzing by generic features, such as the density of numeric characters inside a field, but this approach detects certain types of changes. Interestingly, we address this issue by applying Wrapper Verification and maintenance techniques to learn a set of patterns that describe the information that is being extracted from each of the relevant fields. Since the information for even a single field can vary significantly, the system learns the statistical distribution of the patterns for each field. Wrappers can be verified by comparing the patterns of data returned to the learned statistical distribution. When a significant difference is found, an operator can be notified or we can automatically launch the wrapper repair process, which is described in the further section

\section{Volume 6 Issue 7, July 2017 www.ijsr.net}




\section{International Journal of Science and Research (IJSR) \\ ISSN (Online): 2319-7064}

Index Copernicus Value (2015): 78.96 | Impact Factor (2015): 6.391

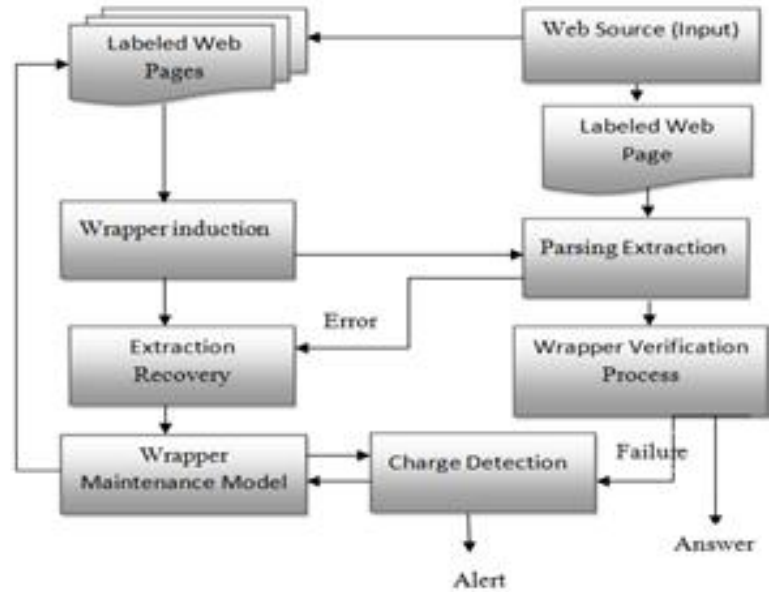

\section{A. Input Web Source}

Web information extraction utilizes the automation of website page get to, restriction and extraction. Module used to outline the capacity to make utility to execute various cases of a similar assignment, including the likelihood to click the snap stream of the client, filling frames and selecting menus and buttons, the non-concurring updating of the page, are taken care of by various tools.

\section{B. Data Extraction}

In This module procured information are bundled in the required configuration or format, these data are prepared to be utilized for wrapper verification, this progression is to convey the package, represented to organized information, to a managing System

\section{Wrapper Induction}

Module consists of automatic data labelling for extracted data. Because the latter aspect is described in detail in other work focus the discussion below on the automatic data labelling algorithm. First, DataProG learns the starting and ending patterns that describe the set of training examples. These training examples have been collected during wrapper's normal operation, while it was correctly extracting data from the Web source. The patterns are used to identify possible examples of the data field on the new pages. In addition to patterns, also calculate the mean (and its variance) of the number-of-tokens in the training examples. Each new page is then scanned to identify all text segments that begin with one of the starting patterns and end with one of the ending patterns.

\section{Wrapper Verification}

The primary content-based approach that operating machine learning techniques for wrapper verification was RAPTURE (Happiness). RAPTURE consumes an arrangement of worldwide numeric components to characterize the separated data. These elements are word tally, normal word length and densities of different sorts of token, for example, HTML tokens, accentuation, capitalized letters, and so on. The calculation accepts that these components are irregular factors, taking after a typical circulation whose mean and fluctuation are assessed from the preparation set. Happiness utilizes these appropriations to assess the likelihood that the information removed from the test set take after various appropriations over the element factors. In light of these, aggregate check probability is assessed, which demonstrates whether the wrapper is right or not. In the event that this confirmation likelihood is more prominent than an edge the calculation chooses that the wrapper is right, generally that it is broken. An intriguing perception in the exploratory after effects of RAPTURE is that it accomplishes its best comes about by utilizing HTML thickness as the single numeric component. Including other numerical components lessens altogether its execution.

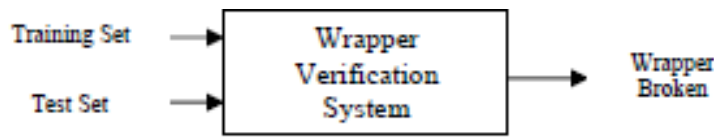

Figure 1: Wrapper System

\section{E. Wrapper Maintenance Model:}

The imagined automated wrapper development and verification process. As indicated by this procedure, the preparation cases are at first gave physically to a wrapper induction, which delivers the principal form of the wrapper. Taking after that point, wrapper confirmation and wrapper re-induction get to be in charge of producing new preparing cases, bringing about enhanced adaptations of the wrapper.

Wrapper authentication is a critical sub-issue of information reconciliation from web sources: one can without much of a stretch envision removing information from many diverse web sources, consuming one wrapper for every each. The capacity to recognize when a wrapper is broken prompts to exceptionally noteworthy investment funds of time and makes wrapper-based information incorporation frameworks suitable. With a specific end goal to represent the previously mentioned ideas we exhibit a basic case of what a wrapper is what's more, why support is required.

\section{Discussion and Result}

We define the following assistant parameters:

$\mathrm{CN}$ : number of correct data items that should be extracted in a page;

EN: number of data items extracted by the wrappers; CEN: number of the correct data items extracted by the wrappers. We use two metrics, Precision and Recall, to evaluate the results of our algorithm of wrapper maintenance. Recall $(\mathrm{R})$ :

proportion of the correctly extracted data items of all the data items that should be extracted.

It can be presented as " $\mathrm{R}=\mathrm{CEN} / \mathrm{CN}$ ". Precision $(\mathrm{P})$ : proportion of the correctly extracted data items of all the data items that have been extracted. It can be presented as " $\mathrm{P}$ $=\mathrm{CEN} / \mathrm{EN} "$.

After applying the first wrappers on the recently gathered pages, the system found those wrappers with low reviews or precisions. We manually checked if the pages had format changes. The results are shown in Table 1. The first column lists the wrapper name. The next two columns list the value of recall (R) and precision (P). Symbol "-" means that the value of $\mathrm{P}$ was not computable. It is on the grounds that the underlying wrappers can't get any information. 


\section{International Journal of Science and Research (IJSR) \\ ISSN (Online): 2319-7064}

Index Copernicus Value (2015): 78.96 | Impact Factor (2015): 6.391

Table 1: Wrapper Maintenance

\begin{tabular}{|c|c|c|c|c|}
\hline Website & R\%(IR) & P\%(IR) & R\%(EX) & P\%(EX) \\
\hline 1Bookstreet Book & 98.67 & 71.26 & 100 & 100 \\
\hline Allbook4less Book & 75 & 32.69 & 75 & 51.34 \\
\hline Amazon Book(search) & 83.05 & 36.3 & 83.05 & 90.74 \\
\hline Amazon Magazine & 100 & 60.15 & 100 & 100 \\
\hline Bamesandnoble & 78.72 & 43.13 & 78.72 & 100 \\
\hline CLA Factbook & 100 & 100 & 100 & 100 \\
\hline CNN Currency & 100 & 100 & 100 & 100 \\
\hline Excite Currency & 100 & 100 & 100 & 100 \\
\hline Hotels Hotel & 50 & 35.61 & 50 & 41.37 \\
\hline Yahoo Shopping & 100 & 51.49 & 100 & 92.86 \\
\hline Yahoo Quotes & 100 & 100 & 100 & 100 \\
\hline Yahoo People & 100 & 53.54 & 100 & 100 \\
\hline \multicolumn{5}{|r}{}
\end{tabular}

Item from the changed pages, thus the value of EN and CEN are all 0 . The forth column provides the number of correct data items of each set of pages considered in this experiment. The last column shows the number of changed pages at the source.

Our work focuses on wrapper construction issue, which incorporates:

1) Building wrappers ,

2) Guaranteeing that the wrappers precisely extract information over a whole collection of pages,

3) Confirming a wrapper to avoid failures when a site changes,

4) and automatically repair wrappers in response to changes in layout or format.

Our principle to resolve all these issue, essentially our approach takes advantage of the fact that web pages have a high degree of regular structure. By analyzing the regular structure of example pages, our wrapper induction process can detect landmarks that enable us to extract desired fields. After developing an initial wrapper induction process we understood that the accuracy of the induction method can be improved by simultaneously learning "forward" and "backward" extraction rules to identify exception cases. Again, what make this possible is the regularities on a page that enable us to identify landmarks both before a field and after a field. Our approach to automatically identifying wrapper breakages and repairing them capitalizes on the regular structure of the extracted fields themselves. Once a wrapper has been initially created, we can use it to obtain numerous examples of the fields. This enables us to profile the information in the fields and obtain structural descriptions that we can use during monitoring and repair.

Essentially this is a bootstrapping approach. Given the early examples provided by the user, we first learn a wrapper. Then we use this wrapper to getting many more examples which we then analyze in much greater depth. Thus by leveraging a few human-provided examples, we end up with a highly scalable system for wrapper creation and maintenance.

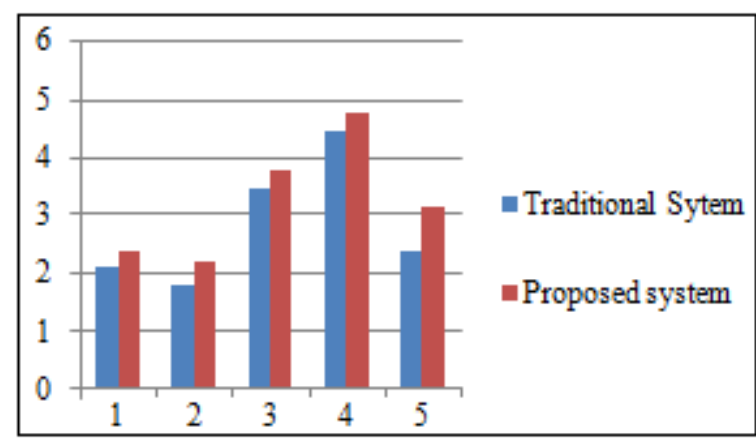

Graph 1: Comparison Traditional system and proposed system for Wrapper change detection rate

Table 1: Comparison Traditional system and Proposed system for Accuracy

\begin{tabular}{|c|c|c|}
\hline Dataset & Traditional Sytem & Proposed system \\
\hline 1 & 2.1 & 2.4 \\
\hline 2 & 1.8 & 2.2 \\
\hline 3 & 3.5 & 3.8 \\
\hline 4 & 4.5 & 4.8 \\
\hline 5 & 2.4 & 3.2 \\
\hline
\end{tabular}

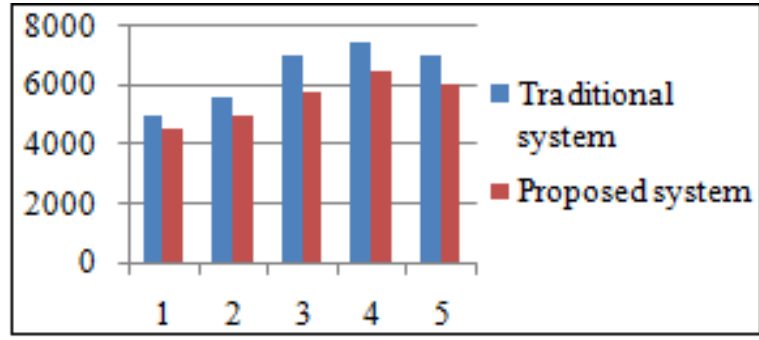

Graph 2: Comparison Traditional system and Proposed system for Execution time

Table 2: Comparison Traditional system and Proposed system for Execution time

\begin{tabular}{|c|c|c|}
\hline Dataset & Traditional system & Proposed system \\
\hline 1 & 5000 & 4500 \\
\hline 2 & 5600 & 5000 \\
\hline 3 & 7000 & 5800 \\
\hline 4 & 7500 & 6500 \\
\hline 5 & 7000 & 6000 \\
\hline
\end{tabular}

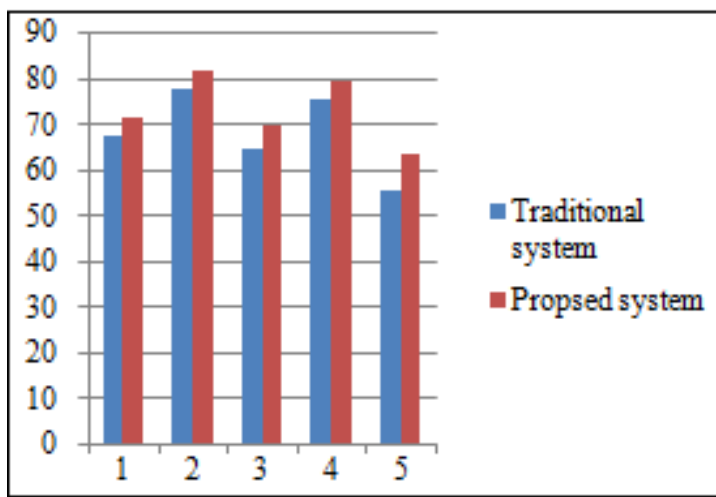

Graph 3: Comparison Traditional system and Proposed system for Accuracy 


\section{International Journal of Science and Research (IJSR) \\ ISSN (Online): 2319-7064}

Index Copernicus Value (2015): 78.96 | Impact Factor (2015): 6.391

Table 3: Comparison Traditional system and proposed system for Accuracy for wrapper change detection.

\begin{tabular}{|c|c|c|}
\hline Websites & Traditional system & Propsed system \\
\hline 1 & 68 & 72 \\
\hline 2 & 78 & 82 \\
\hline 3 & 65 & 70 \\
\hline 4 & 76 & 80 \\
\hline 5 & 56 & 64 \\
\hline
\end{tabular}

From above graph 1 and 2 also table 1and 2 shows comparison of traditional system and proposed system for Recommendation and execution time as recommendation is higher for webpage in proposed system as compared to traditional system and execution time is less for proposed system as compared to traditional system. Third graph shows Accuracy of traditional system is less as compared to proposed system.

\section{Conclusion}

In this paper, we projected the novel approach to wrapper maintenance. The approach uses the effective data features (such as syntactic features, hyperlink, and annotation of extracted data items) that are often preserved after page changes. They are utilized to recognize the areas of the desired data items in the changed pages. Semantic blocks conforming to the user-defined schema are used for grouping data items to recognize the underlying structure of Web pages effectively and precisely. Our intensive experiments with real Web pages showed that our proposed

\section{Acknowledgment}

This work done under the guidance and motivation of Mr. H.P.Khandagale. I would like to give special thanks of gratitude to my guide who gave me an opportunity to do work on the topic. Furthermore, I might want to thanks my parents and friends who supported me, helped me a ton in finishing this work.

\section{References}

[1] Inaki Fernandez de Viana, Pedro J. Abad, Jose Luis Alvarez, and Jose Luis Arjona "MAVE: Multilevel wrApper Verification systEm," IEEETRANSACTIONS ON KNOWLEDGE AND DATA ENGINEERING, VOL. XX, NO. X, SEPTEMBER 2016.

[2] Jozsef Suto, Stefan Oniga, "Comparison of Wrapper and Filter Feature Selection Algorithms on Human Activity Recognition," International Conference on Computers Communications and Control (ICCCC), 2016.

[3] Tony Butler-Yeoman, Bing Xue, and Mengjie Zhang "Particle Swarm Optimisation for Feature Selection: A Hybrid Filter-Wrapper pproach," IEEE Conference on Evolutionary Computation (CEC), pp.2428-2435, 2015.

[4] T. Furche, G. Gottlob, G. Grasso, X. Guo, G. Orsi, C. Schallhart, and C. Wang, "Diadem: Thousands of websites to a single database," Very arge Database Endowment, no. 14, pp. 1845-185, 2014.
[5] E. Ferrara, P. D. Meo, G. Fiumara, and R. Baumgartner, "Web data extraction, applications and techniques: A survey," Knowledge-Based Systems,vol. 70, p. 301323, 2014.

[6] M. Bronzi, V. Crescenzi, P. Merialdo, and P. Papotti,"Extraction and integration of partially overlapping web sources," Very Large Database Endowment, no. 10, pp. 805-516, 2013.

[7] Hassan A. Sleiman , Rafael Corchuelo, "TEX: An efficient and effective unsupervised web information extractor," Knowledge-Based Systems, vol. 39, p. 109123, 2013.

[8] H. A. Sleiman and R. Corchuelo, "A survey on region extractors from web documents," IEEE Transactions on Knowledge and Data Engineering, vol. 99, no. PrePrints, 2012.

[9] Cheng-Ta Wu, Feng Xiang Huang, Kuan Fu Kuo, Ing Jer Huang, "An OCP-AHB Bus Wrapper with Built-in ICE Support for SOC Integration," IEEE Conference,pp.1-4,2012

[10]Filipe Moutinho, Luis Gomes, Aniko Costa, Jose Pimenta, "Asynchronous Wrappers Configurationwithin GALS Systems Specified by Petri Nets," IEEEInternational Conference,pp. 1357 - 1362,2012

[11] Hu Li, Yuanan Liu, Dongming Yuan, Hefei Hu, “A Wrapper of PCI Express with FIFO Interfaces based on FPGA,” International Conference,PP.525-529,2012

[12] N. N. Dalvi, R. Kumar, and M. A. Soliman,"Automatic wrappers for large scale web extraction,"Very Large Database Endowment, no. 4, pp. 805-516,2011.

[13]E. Ferrara and R. Baumgartner, "Design of automatically adaptable web wrappers," in International Conference on Agents and ArtificialIntelligence, 2011, pp. 211-217.

[14] R. B. Emilio Ferrara, "Automatic wrapper adaptation by tree edit distance matchy," Combinations of Intelligent Methods and Application, vol. 20, pp. 41- 53, 2011.

[15] I. F. de Viana, I. Hern'andez, P. Jim'enez, C. R.Rivero, and H. A. Sleiman, "Integrating deep-web information sources," The Distributed Group, Tech.Rep., 2010.

[16]K. Lerman and C. A. Knoblock, "Wrapper maintenance," in Encyclopedia of Database Systems,L. Liu and M. T. Ozsu, Eds. Springer,2009.

[17] S. D. Villalba and P. Cunningham, "An evaluation ofdimension reduction techniques for one-class classification," Artificial Intelligence Review, vol. 27,no. 4, pp. 273-294, 2007

[18]C. D. He X and N. P, "Laplacian score for feature selection," in NIPS: advances in neural information processing systems, 2005, pp. 8-10.

[19]D. Tax, "Ddtools, the data description toolbox formatlab," Dec 2009, version 1.7.3.

[20]C.-H. Chang, M. Kayed, M. R. Girgis, and K. F.Shaalan, "A survey of web information extractionsystems," IEEE Trans. on Knowl. And Data Eng., vol.18, pp. 1411-1428, 2006. 